\title{
The influence of a covariate on optimal designs in longitudinal studies with discrete-time survival endpoints
}

\author{
Maryam Safarkhani*, Mirjam Moerbeek \\ Department of Methodology and Statistics, Utrecht University, The Netherlands
}

\section{A R T I C L E I N F O}

\section{Article history:}

Received 30 October 2012

Received in revised form 12 February 2014

Accepted 12 February 2014

Available online 21 February 2014

\section{Keywords:}

Longitudinal intervention study

Discrete-time survival endpoint

Optimal design

Treatment effect estimator

Covariate effect and prevalence

\begin{abstract}
A B S T R A C T
Longitudinal intervention studies on event occurrence can measure the timing of an event at discrete points in time. To design studies of this kind as inexpensively and efficiently as possible, researchers need to decide on the number of subjects and the number of measurements for each subject. Different combinations of these design factors may produce the same level of power, but each combination can have different costs. When applying a cost function, the optimal design gives the optimal number of subjects and measurements, thus maximizing the power for a given budget and achieving sufficient power at minimal costs. Only very limited research has been conducted on the effect of a predictive covariate on optimal designs for a treatment effect estimator. Here, we go one step further than previous studies on optimal designs and demonstrate the extent to which a binary covariate influences the optimal design. An examination of various covariate effects and prevalences shows how substantially the covariate affects the optimal design and this effect is partly associated with the cost ratio between sampling subjects and measurements, and the survival pattern. So since the optimal design is sensitive to misspecification of these factors, we advise researchers to carefully specify the covariate effect and prevalence.
\end{abstract}

(c) 2014 Elsevier B.V. All rights reserved.

\section{Introduction}

Longitudinal studies follow subjects over a fixed period of time and measure their responses at baseline and multiple successive time points. Studies of this kind are acknowledged as the most powerful tool for identifying the determinants of changes within subjects that occur over time, and as a result they are widely used in many fields of science. In medical science, for example, they are used to study the causes of death or the course of diseases. In social and behavioral sciences, they are commonly used to study changes in attitudes, opinions and behavior.

A special type of outcome for longitudinal studies is the survival endpoint. Survival endpoints measure the occurrence and timing of events. The timing of an event is recorded either continuously using precise units or discretely using discrete intervals. In the social sciences, the time to the occurrence of an event cannot always be measured precisely even though the target event may occur at any point in time. For example, if the risk of relapse among recently released alcoholic patients is of interest, researchers who cannot contact the patients every day to record their drinking status may consider discrete intervals for recording the data, for example weekly intervals. Data recorded in discrete intervals are called discrete-time survival data. Discrete-time survival data are also encountered in retrospective studies where memory failure makes it hard to recall the exact timing of the event, or in studies where the events can only occur at discrete time points. For example, there are only a few time points in a year when high school students can graduate and enter college.

\footnotetext{
* Correspondence to: Department of Methodology and Statistics, Utrecht University, PO Box 80140, 3508 TC Utrecht, The Netherlands. Tel.: +31 30 2534911; fax: +31302535797.

E-mail addresses: M.Safarkhani@uu.nl (M. Safarkhani), M.Moerbeek@uu.nl (M. Moerbeek).
} 
Furthermore, longitudinal studies with discrete-time survival outcomes are commonly applied in experimental settings to compare the measurements of treatment and control groups. Longitudinal intervention studies need to be carefully designed so as not to waste money and time or reduce the subjects' willingness to participate. The primary objective is to detect differences between various treatment groups with adequate statistical power. The power to detect these differences logically depends on the number of subjects in the studies. An important step in designing longitudinal studies is thus to determine the power and avoid two serious miscalculations in sample sizes: an insufficient sample size can lead to inadequate statistical power, whereas an unnecessarily large sample size might be a waste of the researchers' time and money.

Authors such as Raudenbush and Liu (2001), Liu et al. (2000), Hedeker et al. (1999) and Yi and Panzarella (2002) note that researchers not only need to determine the number of subjects, but also the number of repeated measurements for each subject. The power increases if there are more subjects or measurements. However, in situations where researchers are unable to sample as many eligible subjects as needed, increasing the number of measurements may be an extra burden for the subjects and increase the risk of dropout. Moreover, increasing any of these factors can make the study more expensive.

Different numbers of subjects or numbers of measurements can produce the same level of power at different costs. Costs are associated with sampling subjects and taking repeated measurements. Since research budgets are never unlimited, researchers need to consider cost restrictions at the design stage. The best way to design longitudinal intervention studies is to compute optimal designs, i.e. the best combination of the numbers of subjects and measurements so a sufficient power level is achieved at a minimal cost or the power level is maximized for a given budget.

Optimal designs at the lowest cost have been examined in longitudinal studies with various outcomes. Galbraith and Marschner (2002), Passos et al. (2011) and Moerbeek (2008) report the optimal design for growth models with continuous outcomes and Tekle et al. (2008) study the optimal design for trials with dichotomous outcomes. Moreover, Jóźwiak and Moerbeek (2012) examine the optimum combination of a number of subjects and number of time periods for studies with discrete-time survival responses.

Applied researchers have sought an alternative method to increase power and efficiency without increasing the sample size or number of measurements. Research results show that the use of a strongly predictive covariate increases power to some extent (see, e.g., Allison, 1995; Allison et al., 1997). A recent investigation of this method in longitudinal intervention studies with discrete-time survival endpoints is available in Safarkhani and Moerbeek (2013). They demonstrate how much the covariate effect size and covariate prevalence influence the power to detect a treatment effect. What we do not know yet is whether the use of a covariate affects optimal designs for a treatment effect estimator and if so, to what extent. Despite a wide range of studies on optimal designs in longitudinal intervention studies, to the authors' knowledge none of them examine the effect of a covariate effect size and prevalence on optimal designs.

Our aim here is to determine the extent to which a binary covariate influences the optimal numbers of subjects and time points. This paper focuses on longitudinal intervention studies with discrete-time survival endpoints and two treatment groups of equal size where the main objective is to compare the treatment arms with adequate power. It is logical that longitudinal studies are often affected by dropout if subjects leave the study permanently between any two successive time points due to unforeseen reasons rather than event occurrence. So we develop our study for longitudinal designs with and without allowing for subject attrition. We also apply a maximin procedure to obtain robust designs for the misspecification of model parameter values.

After this introduction, the paper is structured as follows. Section 2 is a brief overview of the logistic regression model for analyzing discrete-time survival data. Then we expand the variance-covariance matrix of parameter estimators given by Jóźwiak and Moerbeek (2012) by incorporating the additional covariate effect parameter in the model. In Section 3, we present cost functions and the optimality criterion for obtaining optimal designs as well as relative efficiency as a measure for comparing alternative designs to the optimal design. The results are given in Section 4. Section 5 gives a hypothetical example that illustrates the use of the covariate for the optimal design. Section 6 discusses the elaboration and future work.

\section{Statistical model: the discrete-time hazard model}

We assess the effect of one treatment relative to a control adjusted for the effect of one binary covariate. In the remainder of this paper, we assume the covariate indicates gender but of course any binary covariate can be used. The trial is viewed as consisting of $N$ subjects and half of them are randomly assigned to the treatment group. The number of females is given by $N \pi$, with the proportion $\pi$ representing the covariate prevalence. We also assume that the subjects enter the study at the same time point and are monitored over $p$ time periods $\left[t_{j-1}, t_{j}\right)$ where $j=1,2, \ldots, p, t_{0}=0$ and $t_{p}=p$. Let $T_{i}$ be a non-negative continuous random variable that measures the survival time for subject $i$ and let a discrete random variable $\Gamma_{i}$ indicate the time interval in which the subject experiences the target event (i.e. $\Gamma_{i}=j$ if $t_{j-1} \leq T_{i}<t_{j}$ ).

The discrete-time hazard probability is used to assess the risk of event occurrence in each discrete time interval. This probability is the conditional probability of a subject experiencing the event in period $j$, given that the event has not occurred in an earlier period. This is expressed by

$$
h\left(t_{j}\right)=P(\Gamma=j \mid \Gamma \geq j)=P\left(t_{j-1} \leq T<t_{j} \mid T \geq t_{j-1}\right)=\frac{S\left(t_{j-1}\right)-S\left(t_{j}\right)}{S\left(t_{j-1}\right)},
$$

where $S\left(t_{j}\right)=P\left(T \geq t_{j}\right)=P(\Gamma>j)$ is the probability that a subject has not yet experienced the event by the end of period $j$. 
To assess the effects of the treatment and covariate on the discrete-time hazard probability, we use the discrete-time hazard model (Singer and Willett, 1993, 2003). This model is a combination of multiple generalized linear models, one for each $p$ time period. Using a logit link function, the model for subject $i$ in time period $j$ is written as

$$
\operatorname{logit} h\left(t_{i j}\right)=\left[\alpha_{1} D_{1 i j}+\alpha_{2} D_{2 i j}+\cdots+\alpha_{p} D_{p i j}\right]+\left[\beta_{1} Z_{i}+\beta_{2} C_{i}\right],
$$

where $D_{k i j}$ for $k=1,2, \ldots, p$ is a time indicator and it is set to 1 in the period it represents and 0 elsewhere. The variable $Z_{i}$ specifies the treatment group and is 0 for subjects in the control group and 1 for those in the experimental group. The variable $C_{i}$ is the binary covariate gender and has the value 0 for males and 1 for females. Both predictor variables are timeinvariant, so subjects are assumed to not change their treatment group or covariate value during the study. Each intercept parameter $\alpha_{k}$ represents the value of the logit hazard probability (the log odds of event occurrence) in time period $k$ for the baseline group. We define the baseline group as the subset of the population with predictors equal to zero, in our case the males in the control group. The slope parameters $\beta_{1}$ and $\beta_{2}$ assess the effect of treatment and gender on the probability of experiencing the event. The model assumes the effects of both the predictors are constant over all the time intervals, i.e. the proportional odds assumption.

Model 2 can be written in matrix notation as

$$
\operatorname{logit}(\mathbf{h}(\mathbf{t}))=\mathbf{X} \theta
$$

where the vector $\mathbf{h}(\mathbf{t})$ contains the hazard probabilities for all the time periods and subjects until they experience the event or drop out of the study or the study concludes $(j=p)$. The vector of unknown parameters $\theta=\left(\alpha_{1}, \alpha_{2}, \ldots, \alpha_{p}, \beta_{1}, \beta_{2}\right)^{\prime}$ has $(p+2)$ elements, and the design matrix $\mathbf{X}$ has $(p+2)$ columns and $\left(\sum_{k=1}^{p} N_{C M_{k}}+\sum_{k=1}^{p} N_{C F_{k}}+\sum_{k=1}^{p} N_{E M_{k}}+\sum_{k=1}^{p} N_{E F_{k}}\right)$ rows. We define $N_{C M_{k}}$ and $N_{C F_{k}}$ as the number of males $(M)$ and females $(F)$ in the control (C) group entering the $k$ th period. Likewise, $N_{E M_{k}}$ and $N_{E F_{k}}$ represent the numbers of males and females in the experimental ( $E$ ) group at risk of event occurrence in time interval $k$.

One method for estimating the vector $\theta$ is via iteratively re-weighted least squares as is extensively described by McCullagh and Nelder (1989). The estimated covariance matrix of $\hat{\theta}$ is given by

$$
\begin{aligned}
\widehat{\operatorname{Cov}(\hat{\theta})}= & \left(\mathbf{X}^{\prime} \hat{\mathbf{W}} \mathbf{X}\right)^{-1}=\left\{\sum_{k=1}^{p} N_{C M_{k}} \hat{W}_{C M_{k}} \mathbf{X}_{C M_{k}}^{\prime} \mathbf{X}_{C M_{k}}+\sum_{k=1}^{p} N_{C F_{k}} \hat{W}_{C F_{k}} \mathbf{X}_{C F_{k}}^{\prime} \mathbf{X}_{C F_{k}}\right. \\
& \left.+\sum_{k=1}^{p} N_{E M_{k}} \hat{W}_{E M_{k}} \mathbf{X}_{E M_{k}}^{\prime} \mathbf{X}_{E M_{k}}+\sum_{k=1}^{p} N_{E F_{k}} \hat{W}_{E F_{k}} \mathbf{X}_{E F_{k}}^{\prime} \mathbf{X}_{E F_{k}}\right\}^{-1},
\end{aligned}
$$

but let us first discuss the elements of the first term at the right side of Eq. (3). The other terms of Eq. (3) are defined in the same way. The vector $\mathbf{X}_{C_{k}}$ for males in the baseline group (i.e. control group) has $(p+2)$ elements with one on the $k$ th element and zeros elsewhere so that the first $p$ elements represent the values on the time period dummies. The $(p+1)$ th and $(p+2)$ th entries take the values on the treatment and covariate. The scalar $\hat{W}_{C_{M_{k}}}$ is the element on the main diagonal of the $N_{C M_{k}} \times N_{C M_{k}}$ estimated weight matrix $\hat{\mathbf{W}}_{C_{M_{k}}}$ computed as $\hat{h}_{C M}\left(t_{k}\right)\left[1-\hat{h}_{C M}\left(t_{k}\right)\right]$, where $\hat{h}_{C M}\left(t_{k}\right)$ is the baseline hazard probability in the $k$ th time interval. $N_{C_{k}}$ determines the number of males in the baseline group who neither experience the event nor drop out of the study in the $(k-1)$ th time interval. For $k \geq 2$ it is given by

$$
\begin{aligned}
N_{C M_{k}} & =N_{C M_{k-1}}\left[1-\hat{h}_{C M}\left(t_{k-1}\right)\right]\left(1-r_{C M}\left(t_{k-1}\right)\right) \\
& =\frac{N(1-\pi)}{2} \hat{S}_{C M}\left(t_{k-1}\right) \Pi_{h=1}^{k-1}\left(1-r_{C M}\left(t_{h}\right)\right),
\end{aligned}
$$

where $r_{C M}\left(t_{h}\right) \in[0,1]$ is the attrition rate and is defined in the next section, and $\hat{S}_{C M}\left(t_{k}\right)=\hat{S}_{C M}\left(t_{k-1}\right)\left[1-\hat{h}_{C M}\left(t_{k}\right)\right]$. One might note that $N_{C M_{1}}=\frac{N(1-\pi)}{2}$ is the number of subjects in the risk set at the beginning of the study.

The vector $\mathbf{X}_{C F_{k}}$ has values one on the $k$ th and $(p+2)$ th elements and zeros elsewhere. Correspondingly, the vector $\mathbf{X}_{E M_{k}}$ has values one on the $k$ th and $(p+1)$ th elements and the vector $\mathbf{X}_{E F_{k}}$ has values one on the $k$ th, $(p+1)$ th and $(p+2)$ th elements and zeros elsewhere.

To detect a significant treatment effect adjusted for the covariate, the $t$-test is applied with a $t$-statistic $t=\left|\frac{\hat{\beta_{1}}}{\operatorname{ase}\left(\hat{\beta}_{1}\right)}\right|$ with $N-1$ degrees of freedom to test the null hypothesis $H_{0}: \beta_{1}=0$ against the alternative $H_{1}: \beta_{1} \neq 0$. Here, the asymptotic standard error ase $\left(\hat{\beta}_{1}\right)$ is the square root of the $(p+1, p+1)$ th entry of $\left.\widehat{\operatorname{Cov}(\hat{\theta}}\right)$ in Eq. (3). The statistical power of a test like this is given by

$$
Z_{1-\gamma}=\left|\frac{\hat{\beta_{1}}}{\operatorname{ase}\left(\hat{\beta_{1}}\right)}\right|-Z_{1-\frac{\alpha}{2}},
$$

where $Z_{1-\gamma}$ and $Z_{1-\frac{\alpha}{2}}$ are the $100(1-\gamma) \%$ and $100\left(1-\frac{\alpha}{2}\right) \%$ standard normal deviates, $\alpha$ is the significant level and $1-\gamma$ is the power. 


\section{Designing studies with and without attrition}

Following Jóźwiak and Moerbeek (2012), we take trials with a maximum duration of $p_{\max }=12$ time intervals into account and a trial of shorter duration is achieved by using a smaller number of time periods $p\left(p=1,2, \ldots, p_{\max }\right)$. Increasing $p$ thus involves increasing the duration of a study. Here, $p_{\max }$ represents the maximum number of time periods a trial can be conducted in. For instance, $p_{\max }$ may be considered the number of months in one year making it possible for subjects to be followed over $1,2, \ldots$ or at most twelve months. In addition, for easy comparison of the hazard probabilities in each period, we assume the time periods are equally spaced and the distance between any set of two adjacent time points is fixed in advance.

We assume the treatment groups are of equal size at baseline and define $\pi$ as the proportion of females. We use stratified randomization in designing trials by stratifying the sample by gender and within each gender, the subjects are randomly assigned to treatment groups such that treatment groups are of equal size. Thus, the total sample size at the beginning of the study is given by $N=N_{C M_{1}}+N_{E M_{1}}+N_{C F_{1}}+N_{E F_{1}}$, where $N_{C M_{1}}=N_{E M_{1}}=\frac{N(1-\pi)}{2}$ and $N_{C F_{1}}=N_{E F_{1}}=\frac{N \pi}{2}$. There are four combinations of treatment group and gender and each of them is called a cell.

The primary objective in experimental settings is to detect the treatment effect with adequate statistical power. Eq. (4) shows that as the treatment effect $\beta_{1}$ increases or its asymptotic standard error ase $\left(\hat{\beta}_{1}\right)$ decreases, the power of the test increases and the estimator $\hat{\beta}_{1}$ becomes more efficient. Moreover, for a given treatment effect, greater power is achieved at the minimum ase $\left(\hat{\beta}_{1}\right)$. The power in longitudinal studies is determined by the sample size $N$ and the number of time periods $p$. A simple way to increase the power and consequently the efficiency of a design is by increasing the sample size $N$ or the number of time points $p$. However, this may increase the costs of collecting data on numerous subjects. It may be advisable to identify the optimal number of subjects $N^{*}$ and time periods $p^{*}$ to achieve the smallest possible value of ase $\left(\hat{\beta}_{1}\right)$ and a highly efficient design. So among all the designs $\xi=(N, p)$, we seek the one $\xi^{*}=\left(N^{*}, p^{*}\right)$ that results in the largest statistical power. In doing so, we study the best combination of $N$ and $p$ for maximizing the power subject to a cost constraint, which is the same as minimizing the cost for a fixed power.

To determine the optimal design, we consider three cost functions that examine various costs of sampling subjects and obtaining measurements for each subject. Cost function I assumes that all the subjects are tracked until the end of a study even if they experience an event in any earlier period. If $C_{s}$ denotes the costs of sampling and recruiting one subject, $C_{m}$ denotes the costs of taking one measurement for each subject, and $C$ is the total budget, then cost function I is defined as

$$
C=N C_{s}+N(p+1) C_{m},
$$

where $N$ is the total sample size at the beginning of the study and $p$ is the number of time periods. The expression in brackets represents the number of measurements for each subject, which adds the number of intervals to one baseline measurement. We assume the costs of measuring the covariate and the costs of implementing the treatment are part of the subject-level costs $C_{s}$.

Cost function II assumes that subjects are not followed after the event occurs and is derived by replacing $p$ in Eq. (5) by

$$
\frac{1-\pi}{2}\left\{\sum_{k=1}^{p} S_{C M}\left(t_{k}\right)+\sum_{k=1}^{p} S_{E M}\left(t_{k}\right)\right\}+\frac{\pi}{2}\left\{\sum_{k=1}^{p} S_{C F}\left(t_{k}\right)+\sum_{k=1}^{p} S_{E F}\left(t_{k}\right)\right\},
$$

where $S\left(t_{k}\right)$ denotes the survival probability at the end of interval $k$ and each subscript of $S$ is used to denote a specific cell. The number of measurements for each subject thus depends on the number of time periods for each subject that varies across subjects.

Cost function III assumes that subjects drop out of the study due to unforeseen reasons such as an unwillingness to participate further or changing jobs before experiencing the event. In practice, longitudinal studies are often harmed by attrition and in addition to various strategies to limit its occurrence, investigators need to take the possibility of attrition into account. So the third cost function is used to investigate optimal designs for studies with attrition and is achieved by replacing $p$ in Eq. (5) by

$$
\begin{gathered}
\frac{1-\pi}{2}\left\{\sum_{k=1}^{p} S_{C M}\left(t_{k}\right) \Pi_{h=1}^{k}\left(1-r_{C M}\left(t_{h}\right)\right)+\sum_{k=1}^{p} S_{E M}\left(t_{k}\right) \Pi_{h=1}^{k}\left(1-r_{E M}\left(t_{h}\right)\right)\right\} \\
+\frac{\pi}{2}\left\{\sum_{k=1}^{p} S_{C F}\left(t_{k}\right) \Pi_{h=1}^{k}\left(1-r_{C F}\left(t_{h}\right)\right)+\sum_{k=1}^{p} S_{E F}\left(t_{k}\right) \Pi_{h=1}^{k}\left(1-r_{E F}\left(t_{h}\right)\right)\right\},
\end{gathered}
$$

where the attrition rate $r_{C M}\left(t_{h}\right)$ denotes the proportion of males in the control group who leave the study during time period $h$ due to reasons other than event occurrence. Correspondingly, $r_{C F}\left(t_{h}\right), r_{E M}\left(t_{h}\right)$ and $r_{E F}\left(t_{h}\right)$ are the attrition rates between the two adjacent time points $h-1$ and $h$ for the other cells. Cost function III is an extension of cost function II, in which the attrition rates are assumed to be zero. In this study, we assume a constant attrition rate across all time periods and all cells, i.e. $r_{C M}\left(t_{h}\right)=r_{E M}\left(t_{h}\right)=r_{C F}\left(t_{h}\right)=r_{E F}\left(t_{h}\right)=r$ for any $h \in\{1,2, \ldots, 12\}$. 
Using Eq. (5), we have

$$
N=\frac{C}{C_{s}+C_{m}(p+1)}=\frac{C}{C_{m}(f+p+1)},
$$

where $f=\frac{C_{s}}{C_{m}}$ is the cost ratio. Similarly, sample size $N$ can be calculated for the second and third cost functions by replacing $p$ by the corresponding summation of survival probabilities.

We look for the optimal design $\xi^{*}=\left(N^{*}, p^{*}\right)$ so that an optimality criterion is minimized. We define the optimality criterion as the variance of the estimator of a regression coefficient, $\operatorname{var}\left(\hat{\beta}_{1}\right)$ and $/$ or $\operatorname{var}\left(\hat{\beta}_{2}\right)$. If the primary objective of a study is to estimate the treatment effect $\beta_{1}$ as efficiently as possible, then a single-objective optimal design minimizes var $\left(\hat{\beta_{1}}\right)$.

To compare the efficiency of design $\xi$ relative to the optimal design $\xi^{*}$ for the criterion $\operatorname{var}\left(\hat{\beta}_{1}\right)$, the relative efficiency $(\mathrm{RE})$ is used as in

$$
\mathrm{RE}_{\xi \mid \xi^{*}}=\frac{\operatorname{var}\left(\hat{\beta}_{1}^{*}\right)}{\operatorname{var}\left(\hat{\beta}_{1}\right)},
$$

where the RE also enables us to determine how many subjects are needed so that a design $\xi$ has the same efficiency as the optimal design $\xi^{*}$. If $N_{\xi *}$ subjects are needed for the optimal design $\xi^{*}$, we obtain the same efficiency by using $N_{\xi}=N_{\xi} / R_{\xi} \xi^{*}$ subjects from design $\xi$. RE values close to 1 are thus preferable.

\subsection{Weibull survival function}

Since the underlying surviving time variable $T$ is assumed to be continuous, different continuous-time survival functions can be used to describe its probability density function such as Exponential, Weibull, Lognormal, Gamma or Gumbel. Since it represents constant, decreasing and increasing hazard functions, the Weibull distribution function is a common and flexible model for this purpose. The Weibull function with parameters $\tau$ and $\lambda$ is expressed as $f(t)=\lambda \tau t^{\tau-1} \exp \left(-\lambda t^{\tau}\right)$. Here, $t \in[0,1]$ represents the proportion of time elapsed in the study so that 0 refers to the beginning $\left(t_{0}\right)$ and 1 to the end of the study with maximum duration $\left(t_{p_{\max }}\right)$. The Weibull survival function is thus given by $S(t)=e^{-\lambda t^{\tau}}$ and the corresponding baseline hazard function by $h(t)=\lambda \tau t^{\tau-1}$. The scale parameter $\lambda$ can be replaced by $-\log (1-\omega)$ with $\omega \in[0,1]$ as the overall proportion of the subjects in the baseline group who experience the event by time $t=1$ (Galbraith and Marschner, 2002). The shape parameter $\tau \in[0, \infty)$ determines the shape of the hazard function. If $\tau<1$, the hazard function decreases over time and represents a study with many subjects experiencing the event at the beginning of the study. If $\tau=1$, the hazard function is constant over the course of the study. A value of $\tau>1$ specifies an increasing hazard function and implies the highest risk of event occurrence towards the end of the trial. Given Weibull survival parameters $\omega$ and $\tau$, we compute the survival probability $S\left(t_{j}\right)$ at the end of interval $j$ with $t_{j}=\frac{j}{p_{\max }}$. We also calculate the baseline discrete-time hazard probability $h\left(t_{j}\right)$ by considering Eq. (1). We specify the hazard probabilities for the other subgroups by adding the parameters $\beta_{1}$ and $\beta_{2}$ on the logit scale to the baseline hazard probability. This implies that the survival functions are not Weibull in the other subgroups rather than the baseline.

\section{Results}

\subsection{The effect of the covariate effect and prevalence}

In general, our results show that a design that has high efficiency for the estimator of $\beta_{1}$ also performs reasonably well for the estimator of $\beta_{2}$. The estimator of $\beta_{1}$ is considered the more important objective in optimal designs, but we also present results of the RE for the estimator of $\beta_{2}$ because it might be of secondary interest. We investigate the extent to which the covariate effect $\beta_{2}$ and the covariate prevalence $\pi$ change the optimal design for $\operatorname{var}\left(\hat{\beta_{1}}\right)$. Results are solely presented for trials where subjects only leave the study after experiencing the event of interest (cost function II). Figures for the other cost functions can be found in the supplementary material (see Appendix A).

We discuss our results for a specific combination of design factors given by $\tau=1, f=1, \omega=0.5$ and $\beta_{1}=1.5$. This combination characterizes trials with a constant baseline hazard function where $50 \%$ of the subjects in the baseline group have experienced the event by the end of time interval $p_{\max }$. In addition, the subject-level costs $C_{s}$ are as high as the measurement-level costs $C_{m}$ and the odds ratio of event occurrence is 4.5 times as high for the experimental group as for the control group (i.e. $\beta_{1}=1.5$ ). Fig. 1 depicts the RE of both the optimality criteria as a function of the number of time periods $p$ (on the horizontal axis in each panel), the covariate effect $\beta_{2}$ (separate panels in each column) and the covariate prevalence $\pi$ (separate panels in each row). In each panel, the solid line represents the RE of $\operatorname{var}\left(\hat{\beta}_{1}\right)$ and the dashed line shows the RE of $\operatorname{var}\left(\hat{\beta}_{2}\right)$. The dotted horizontal lines indicate RE values of 0.8 and 1 and the dotted vertical line shows the optimal number of time periods giving the smallest $\operatorname{var}\left(\hat{\beta}_{1}\right)$.

We first explain the effect of the covariate prevalence $\pi$ on the optimal design. As can be deduced from Fig. 1, an increase in $\pi$ leads to a higher optimal number of time periods if $\beta_{2}=-1.5$, but $\pi$ does not affect the optimal design if $\beta_{2}=0$. If 

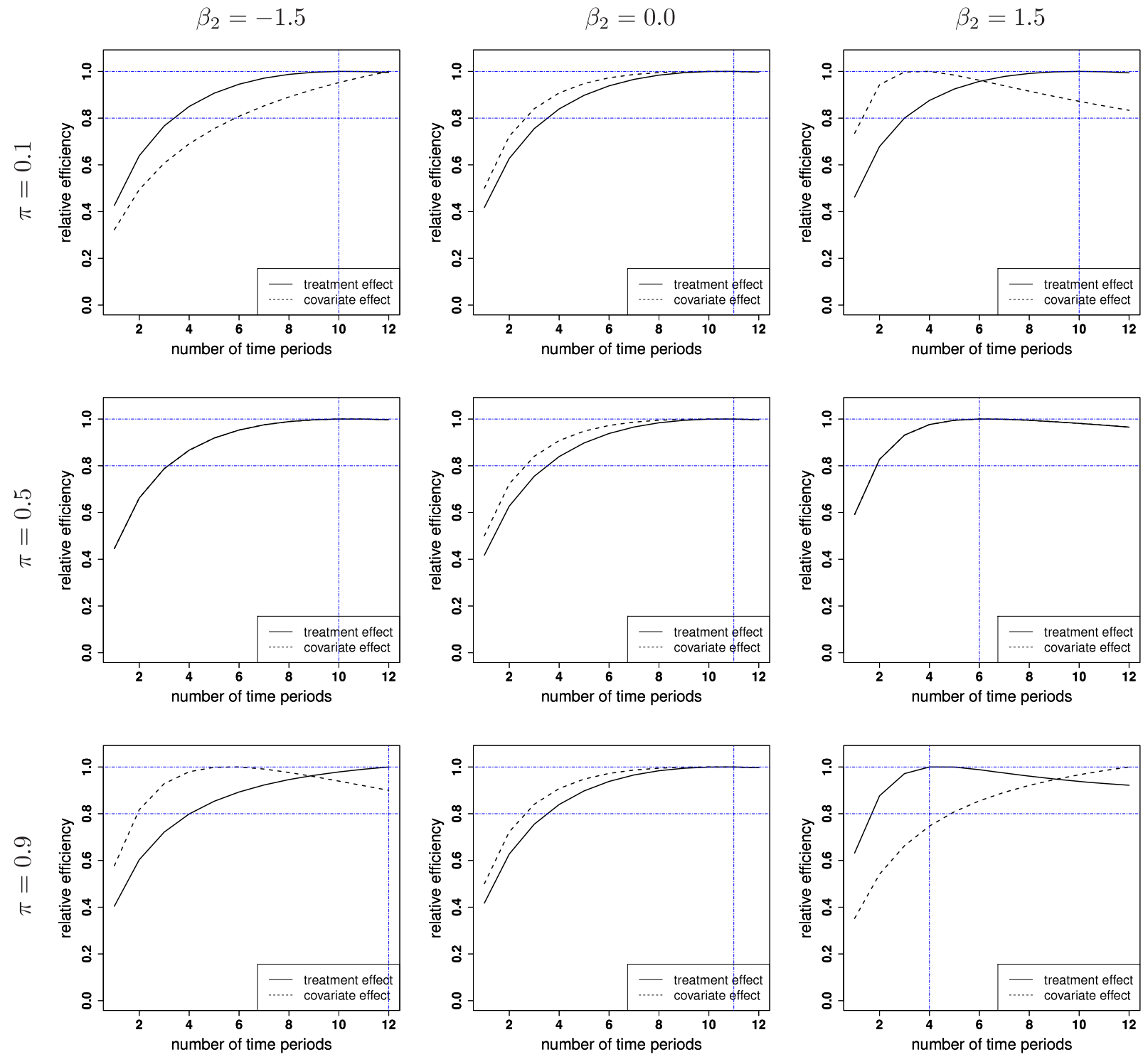

Fig. 1. Efficiency plots for the treatment and covariate effects as a function of the number of time periods, $\beta_{2}$ and $\pi$ with $\tau=1, f=1, \omega=0.5$ and $\beta_{1}=1.5$.

$\beta_{2}=+1.5$, the optimal number of time periods decreases as $\pi$ increases. If $\beta_{2} \neq 0$, the two RE curves for $\operatorname{var}\left(\hat{\beta}_{1}\right)$ and $\operatorname{var}\left(\hat{\beta}_{2}\right)$ overlap if $\pi=0.5$. The reason is that since the four cells are of equal size at baseline if $\pi=0.5$, they are of equal size in all the periods if $\beta_{1}=\beta_{2}$. So the variables $Z$ and $C$ in Eq. (2) are interchangeable and then $\operatorname{var}\left(\beta_{1}\right)=\operatorname{var}\left(\beta_{2}\right) \forall p \in\{1,2, \ldots, 12\}$. As coding the variables $Z$ and $C$ is arbitrary, the two variables are still interchangeable if $\beta_{1}=-\beta_{2}$, and for the same reason $\operatorname{var}\left(\beta_{1}\right)=\operatorname{var}\left(\beta_{2}\right) \forall p \in\{1,2, \ldots, 12\}$.

We now focus on the effect of the covariate effect $\beta_{2}$ on the optimal design. We observe that increasing $\beta_{2}$ from -1.5 to +1.5 has a minimal effect on the optimal number of time periods if $\pi=0.1$, but this effect becomes stronger as $\pi$ increases so that the optimal number of time periods substantially decreases with increasing $\beta_{2}$ if $\pi=0.9$. In some plots (e.g. the left-upper plot in Fig. 1), the RE curve may be flat and close to 1 across a few time periods, although numerically the RE is only equal to 1 at one time period. In cases like this, any number of time periods in that range could be chosen as having a sufficiently efficient design if designing the study with the optimal value of $p$ is not feasible in practice.

We also investigate how the effect of $\beta_{2}$ and $\pi$ on the optimal number of time periods depends on the cost ratio $f$ and survival pattern $\tau$ (figures not shown). We observe that the optimal number of time periods does not depend on $\beta_{2}$ and $\pi$ if sampling a subject becomes more expensive than obtaining a repeated measurement for each subject $(f=10,100)$. The optimal number of time periods leans towards larger values in the range of $\{1,2, \ldots, 12\}$ if the hazard function increases over time $\left(\tau>1\right.$ ), so that a less sizable effect of $\beta_{2}$ and $\pi$ on the optimal design is observed if $\tau>1$ compared to $\tau<1$ or $\tau=1$. 


\section{Table 1}

Optimal number of time points $p^{*}$ as a function of the covariate effect $\beta_{2}$ and prevalence $\pi$ and for various cost functions.

\begin{tabular}{lllll}
\hline Cost function & Covariate prevalence & \multicolumn{3}{l}{ Covariate effect } \\
\cline { 3 - 5 } & & $\beta_{2}=-1.5$ & $\beta_{2}=0.0$ & $\beta_{2}=+1.5$ \\
\hline I & $\pi=0.1$ & 5 & 5 & 4 \\
& $\pi=0.5$ & 6 & 5 & 3 \\
II & $\pi=0.9$ & 9 & 5 & 2 \\
& $\pi=0.1$ & 10 & 11 & 10 \\
III $(r=0.25)$ & $\pi=0.5$ & 10 & 11 & 6 \\
& $\pi=0.9$ & 12 & 11 & 4 \\
& $\pi=0.1$ & 12 & 12 & 12 \\
& $\pi=0.5$ & 12 & 12 & 10 \\
& $\pi=0.9$ & 12 & 12 & 5 \\
\hline
\end{tabular}

Note. $\tau=1, f=1, \omega=0.5$ and $\beta_{1}=1.5$.

\subsection{The effect of the cost function}

Thus far, we have only focused on cost function II. In this section, we study the extent to which $\beta_{2}$ and $\pi$ affect the optimal design if the other cost functions are considered. Table 1 presents the optimal number of time points $p^{*}$ as a function of the covariate effect $\beta_{2}$ and the covariate prevalence $\pi$ for different cost functions for the same combination of design factors $\left(\tau, f, \omega\right.$ and $\beta_{1}$ ) as in Fig. 1. In general, we observe two main findings also observed by Jóźwiak and Moerbeek (2012). Firstly, the optimal number of time points is larger for cost function II or III. Secondly, the optimal number of time points with cost function II is somewhat smaller than or equal to those with cost function III. So attrition may slightly influence the optimal design. We now compare the effects of $\beta_{2}$ and $\pi$ on the optimal number of time points $p^{*}$ between the cost functions, and first discuss the effect of $\pi$ for a given $\beta_{2}$. If $\beta_{2}=-1.5$, the increase of $\pi$ results in a much larger increase in $p^{*}$ if cost function I is used instead of cost function II or III. However, if $\beta_{2}=+1.5, p^{*}$ decreases as $\pi$ increases, this effect is more noticeable for cost function II or III. We now discuss whether the effect of $\beta_{2}$ on $p^{*}$ changes when using different cost functions for a given $\pi$. As $\beta_{2}$ increases, the optimal number of time points $p^{*}$ mainly decreases, and all the more so with a larger $\pi$, regardless of which cost function is used. The cost function thus hardly influences the effect of $\beta_{2}$ on the optimal design.

It should be noted that we only include trials with a constant hazard function $(\tau=1)$ and an equal cost ratio $(f=1)$ in Table 1. We assess the effect of the cost function on the relation between $\beta_{2}$ and $p^{*}$ for different cost ratios and survival patterns (tables not shown). The decrease in $p^{*}$ with an increase in $\beta_{2}$ is smaller for cost function II or III if $f \gg 1$. Based on a decreasing hazard function, $p^{*}$ steadily decreases if $\beta_{2}$ increases with cost function I, while the relation appears to be curvy or reversed with other cost functions. Moreover, in trials with an increasing $(\tau>1)$ or a constant $(\tau=1)$ hazard function, the effect of $\beta_{2}$ on the decrease in $p^{*}$ is more substantial with cost function I than the other cost functions.

\subsection{Maximin optimal designs}

The variance-covariance matrix in Eq. (3) and consequently the optimality criterion $\operatorname{var}\left(\hat{\beta}_{1}\right)$ depends on the unknown parameters $\beta_{1}, \beta_{2}, \omega, \tau$ and $r$ and thus requires initial and reliable estimates based on a pre-study, either that or educated guesses have to be made in advance. Good initial estimates of the values of the other parameters are not always available in practice. In these cases, calculating the optimal design might be problematic since the initial estimates are poor. We consider a maximin optimal design criterion to find more robust designs in a pre-specified region of the parameter values. This criterion requires an a priori specification of a plausible region for the model parameters. In this paper, we confine ourselves to the parameters $\beta_{1}$ and $\beta_{2}$ and assume that researchers have an idea about the cost ratio $f$, the underlying survival parameters $\omega$ and $\tau$, and the attrition rate $r$. Suppose $\left(\beta_{1}, \beta_{2}\right) \in \mathbf{B}$ such that $\mathbf{B}=\mathbf{B}_{\mathbf{1}} \times \mathbf{B}_{\mathbf{2}}=\left[\beta_{1_{L}}, \beta_{1_{U}}\right] \times\left[\beta_{2_{L}}, \beta_{2_{U}}\right] \subset R^{2}$ is a sub-region of the parameter space, and let $\Xi$ represent the set of all the possible designs $\xi=(N, p)$. A design $\xi^{\text {maximin }}$ is referred to as the maximin design for estimating $\beta_{1}$ if it maximizes a set of smallest REs over $\Xi$ (Berger and Wong, 2009, Section 5.3.1.1). The maximin design performs best in terms of the minimal RE and the corresponding maximin efficiency is

$$
\operatorname{MME}=\max _{\xi \in \Xi}\left\{\min _{\left(\beta_{1}, \beta_{2}\right) \in \mathbf{B}}\left[\operatorname{RE}\left(\xi, \xi^{*}\right)\right]\right\},
$$

where for each vector of $\left(\beta_{1}, \beta_{2}\right) \in \mathbf{B}$, the relative efficiency $\operatorname{RE}\left(\xi, \xi^{*}\right)$ is computed with respect to the locally optimal design $\xi^{*}$ for the particular values of $\beta_{1}$ and $\beta_{2}$. We assume an initial estimate of +1.5 for the treatment effect as well as the covariate effect $\left(\beta_{1_{0}}=\beta_{2_{0}}=+1.5\right)$, and specify different plausible regions around these initial estimates reflecting the degree of uncertainty about them. Table 2 shows the number of time periods of the maximin optimal designs $\left(p^{\text {maximin }}\right)$ for different covariate prevalences if $f=1, \tau=1$ and $\omega=0.5$. The numbers in parentheses are the MMEs and the corresponding sample size $N^{\text {maximin }}$ is obtained by replacing $p^{\text {maximin }}$ in cost function II. Each row represents a region for the initial value $\beta_{1_{0}}$, with the first one reflecting very little uncertainty in $\beta_{1_{0}}$ and the last one reflecting the greatest uncertainty. In addition, each column represents a region for the initial value $\beta_{2_{0}}$; the first region is narrower than the next ones.

In general, we observe that $p^{\text {maximin }}$ is often smaller than or equal to $p^{\text {locally }}$, and the MME is always smaller than 1 so that applying the maximin design leads to a loss in efficiency. The loss in efficiency is larger if one or both regions $\mathbf{B}_{\mathbf{1}}$ and $\mathbf{B}_{\mathbf{2}}$ are 
Table 2

Maximin number of time periods $p^{\text {maximin }}$ for estimation of $\beta_{1}$ for various choices of $\mathbf{B}=$ $\left[\beta_{1_{L}}, \beta_{1_{U}}\right] \times\left[\beta_{2_{L}}, \beta_{2_{U}}\right]$ and $\pi$ with $\tau=1, f=1, \omega=0.5$.

\begin{tabular}{lllll}
\hline Covariate prevalence & $\mathbf{B}_{\mathbf{1}}=\left[\beta_{1_{L}}, \beta_{1_{U}}\right]$ & \multicolumn{3}{l}{$\mathbf{B}_{\mathbf{2}}=\left[\beta_{2_{L}}, \beta_{2_{U}}\right]$} \\
\cline { 3 - 5 } & & {$[+1.4,+1.6]$} & {$[+1.2,+1.8]$} & {$[+1.0,+2.0]$} \\
\hline$\pi=0.1$ & {$[+1.4,+1.6]$} & $11(0.996)$ & $11(0.994)$ & $11(0.991)$ \\
$\pi=0.5$ & {$[+1.2,+1.8]$} & $10(0.977)$ & $10(0.975)$ & $10(0.971)$ \\
& {$[+1.0,+2.0]$} & $10(0.947)$ & $10(0.945)$ & $10(0.940)$ \\
$\pi=0.9$ & {$[+1.4,+1.6]$} & $4(0.980)$ & $5(0.968)$ & $10(0.949)$ \\
& {$[+1.2,+1.8]$} & $3(0.965)$ & $4(0.932)$ & $5(0.903)$ \\
& {$[+1.0,+2.0]$} & $3(0.940)$ & $4(0.891)$ & $4(0.866)$ \\
& {$[+1.4,+1.6]$} & $3(0.941)$ & $6(0.914)$ & $7(0.883)$ \\
& {$[+1.2,+1.8]$} & $2(0.917)$ & $3(0.833)$ & $4(0.796)$ \\
& {$[+1.0,+2.0]$} & $1(0.891)$ & $2(0.793)$ & $3(0.727)$
\end{tabular}

Note. Given are the $p^{\text {maximin }}$ and the corresponding MME in parentheses, and the designs were found by searching over a grid of 0.01 in $\mathbf{B}$.

wider than if both regions are narrow. However, the degree to which the loss in efficiency increases as $\mathbf{B}_{\mathbf{1}}$ or $\mathbf{B}_{\mathbf{2}}$ becomes wider depends on the value of $\pi$, and it is larger if $\pi$ is larger. In addition, specifying more diffuse or less diffuse regions $\mathbf{B}_{\mathbf{1}}$ and $\mathbf{B}_{\mathbf{2}}$ somewhat changes $p^{\text {maximin }}$ if $\pi=0.1$, and it greatly influences $p^{\text {maximin }}$ if $\pi$ is larger. In these cases, $p^{\text {maximin }}$ increases as $\mathbf{B}_{\mathbf{2}}$ becomes wider for a given $\mathbf{B}_{\mathbf{1}}$, which is stronger for a narrower $\mathbf{B}_{\mathbf{1}}$, and $p^{\text {maximin }}$ decreases as $\mathbf{B}_{\mathbf{1}}$ becomes wider for a given $\mathbf{B}_{2}$, which is stronger for a wider $\mathbf{B}_{\mathbf{2}}$. Lastly, $p^{\text {maximin }}$ and MME generally become smaller if $\pi$ increases for a given combination of $\mathbf{B}_{\mathbf{1}}$ and $\mathbf{B}_{\mathbf{2}}$. This means if a researcher has no information on the true $\beta_{1}$ and $\beta_{2}$ values, the loss in efficiency using maximin designs is at most $6 \%$ if $\pi=0.1$ and at most $13 \%$ if $\pi=0.5$ and $27 \%$ if $\pi=0.9$, suggesting that the maximin design will require many more subjects to achieve the same efficiency as the locally optimal design if $\pi$ is large.

We also studied the maximin designs for different regions of the initial estimates $\beta_{2_{0}}=0$ and $\beta_{2_{0}}=-1.5$ (tables are in the supplementary material, see Appendix A). For $\beta_{2_{0}}=0, p^{\operatorname{maximin}}$ does not depend on $\pi$ or on the regions $\mathbf{B}_{\mathbf{1}}$ and $\mathbf{B}_{\mathbf{2}}$, and is not much larger than $p^{\text {locally }}$. The more diffuse the $\mathbf{B}_{\mathbf{1}}$ or $\mathbf{B}_{\mathbf{2}}$, the greater the loss in efficiency, especially if $\pi$ is large. This point is striking if $\beta_{2_{0}}=-1.5$ and less so if $\beta_{2_{0}}=0$ or $\beta_{2_{0}}=+1.5$, so that with only more restrictive regions, the maximin design is still efficient (MME $>0.80$ ) as $\pi$ increases. More reliable information is thus needed to specify regions for $\beta_{1}$ and $\beta_{2}$ values if the covariate prevalence is high or a negative covariate effect is presumed possible for the study.

\section{Application: the effect of parental transition on early sexual intercourse}

Its connection to unintended teenage pregnancy and sexually transmitted disease including HIV infection makes adolescent sexuality an ever present social concern. Given the need to prevent early heterosexual intercourse, numerous studies have examined factors associated with the timing of the early initiation of intercourse. Capaldi et al. (1996) investigate the risk of initial heterosexual intercourse in a sample of 180 at-risk boys $(N=180)$. Among the subjects followed from the 7 th grade, $30 \%$ were still virgins when the study ended in the 12th grade. The authors also focus on the effect of parental transitions (PT) prior to the 7th grade on the risk of initial adolescent intercourse $(\pi=0.6)$. Fig. 2 shows the estimated logit hazard and survival probabilities as a function of the grade at initial heterosexual intercourse for each group separately. Only a few subjects initiate sex at earlier ages but as time progresses the number of subjects who remain at risk steadily decreases. The hazard function does not steadily decline or rise over time and hence does not correspond to the Weibull hazard function. So this example shows how our method can be used for any trial without the assumption that the underlying survival function is Weibull. In addition, the effect of PT is estimated as $\beta_{2}=+0.8736$, which means the estimated odds of initially having intercourse are 2.5 times as high for boys who experience a parental transition as for those who do not (see Table 11.3 in Singer and Willett (2003)).

Research on adolescent sexuality tends to promote effective sex education and intervention programs designed to help adolescents develop decision-making and communication skills, and increase knowledge about disease transmission and prevention. A similar longitudinal study by Capaldi et al. (1996) aims to study the effect of intervention programs on delaying initial intercourse. We assume that teenagers who have attended a program engage in early sexual activity at a lower risk $\left(\beta_{1}=-0.5\right)$. The $\beta_{1}$ value corresponds to an increase of about $15 \%$ in the survival probability of remaining virgins in either PT group by the end of the study. We consider this a reasonable effect size for an effective program. Our primary interest is to select the best combination of number of subjects $N$ and number of time intervals $p$ to estimate the program effect $\beta_{1}$ as precisely as possible. We also want to clarify the effect of PT on the optimal combination of $N$ and $p$. To do so, we consider other PT effect size values such as $\beta_{2}=-0.8736$ and $\beta_{2}=0$ to illustrate how the optimal design for $\operatorname{var}\left(\hat{\beta}_{1}\right)$ is sensitive to an incorrectly specified $\beta_{2}$. We assume that recruiting a new subject is three times more expensive than taking one measurement for each subject $(f=3)$, and compute the costs of the study using cost function II.

Table 3 gives the power level for trials with 180 adolescents followed over $1,2, \ldots$ or six school grades. In addition, the table shows the required sample size to achieve a power level of 0.8 and the associated total costs for each trial with a different number of time points $p$ and different $\beta_{2}$ values. If we follow a sample of 180 adolescents at six equidistant time 

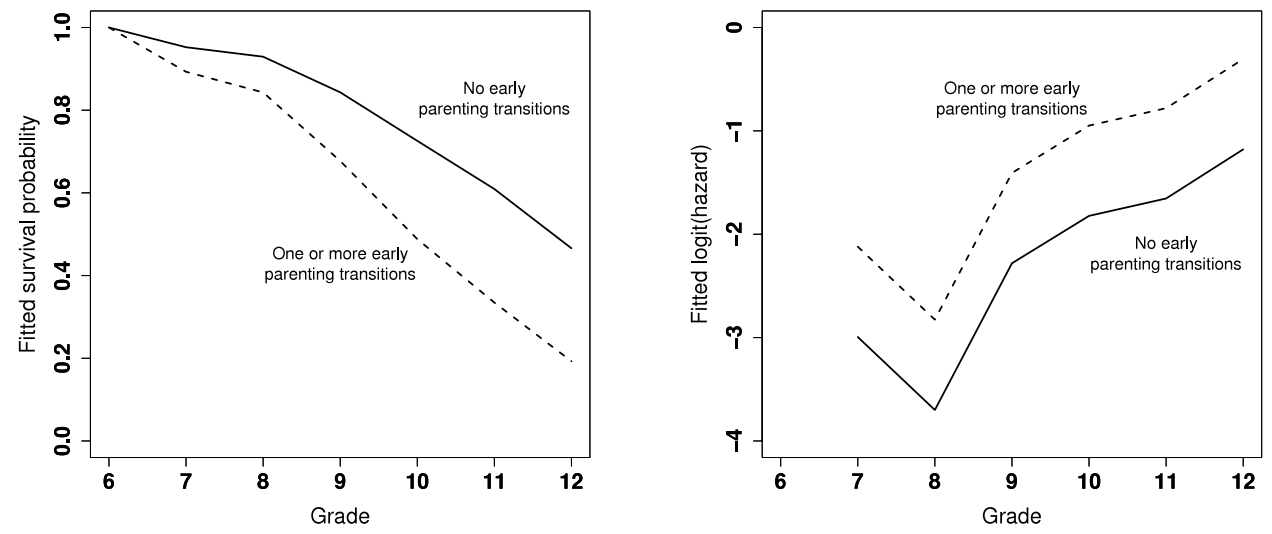

Fig. 2. Fitted survivor function (left side) and logit (hazard) function (right side) for the grade at first intercourse data.

Table 3

Optimal design for the grade at first intercourse data.

\begin{tabular}{|c|c|c|c|c|c|c|c|c|c|}
\hline \multirow[t]{2}{*}{ Number of time periods $(p)$} & \multicolumn{3}{|c|}{ Power with $N=180$} & \multicolumn{3}{|c|}{$N$ to get power 0.8} & \multicolumn{3}{|c|}{ Total cost of the study ${ }^{\mathrm{a}}$} \\
\hline & $\begin{array}{l}\beta_{2}= \\
-0.87\end{array}$ & $\begin{array}{l}\beta_{2}= \\
0.00\end{array}$ & $\begin{array}{l}\beta_{2}= \\
+0.87\end{array}$ & $\begin{array}{l}\beta_{2}= \\
-0.87\end{array}$ & $\begin{array}{l}\beta_{2}= \\
0.00\end{array}$ & $\begin{array}{l}\beta_{2}= \\
+0.87\end{array}$ & $\begin{array}{l}\beta_{2}= \\
-0.87\end{array}$ & $\begin{array}{l}\beta_{2}= \\
0.00\end{array}$ & $\begin{array}{l}\beta_{2}= \\
+0.87\end{array}$ \\
\hline 1 & 0.07 & 0.09 & 0.13 & 5409 & 3578 & 2103 & 269.1 & 177.5 & 103.7 \\
\hline 2 & 0.09 & 0.12 & 0.17 & 3621 & 2395 & 1409 & 215.0 & 141.4 & 82.2 \\
\hline 3 & 0.15 & 0.21 & 0.30 & 1620 & 1090 & 685 & 111.0 & 73.9 & 45.3 \\
\hline 4 & 0.24 & 0.32 & 0.44 & 912 & 628 & 427 & 70.2 & 47.4 & 31.0 \\
\hline 5 & 0.32 & 0.43 & 0.56 & 625 & 439 & 320 & 52.9 & 36.1 & 24.9 \\
\hline 6 & 0.43 & 0.55 & 0.65 & 444 & 323 & 257 & 40.6 & 28.3 & 21.0 \\
\hline
\end{tabular}

Note. $f=3, \pi=0.6, \omega=0.7$ and $\beta_{1}=-0.5$ in cost function II.

${ }^{\text {a }}$ Total cost is multiplied by 100 and $C_{m}$ is set to 1 .

points, the power levels are poor for any $\beta_{2}$. To obtain a power level of 0.8 , we need to increase $N$ by including 264,143 or 77 additional subjects, depending on $\beta_{2}$. The table shows other possible combinations of $N$ and $p$ to get $80 \%$ power. To select the designs $\xi=(N, p)$ worth taking, we compare their corresponding costs. The most cost-efficient design is the one with only a few subjects observed at six time points, and this holds true for each value of $\beta_{2}$. Although in this example, $\beta_{2}$ does not influence the optimal design and the optimal design is always the one with the maximum number of time periods, $\beta_{2}$ influences the sample size and consequently the cost of the study. If one erroneously selects -0.8736 as the PT effect size, one will recruit many more subjects and in turn design a less cost-efficient study.

It should be noted that there is no nesting of boys in classes and schools in the calculation of the optimal design. To avoid the risk of control group contamination, schools rather than boys may be randomized to treatment groups. A cluster randomized trial requires a larger sample size and multilevel analysis. Only limited research has been conducted on multilevel models for discrete-time survival analysis. Moerbeek (2012) has recently studied the relation between sample size and power for cluster randomized trials, but does not take covariates into account. The cost-efficiency of adding covariates to cluster randomized trials with continuous outcomes is discussed by Moerbeek (2006).

\section{Conclusion and a discussion}

The overall results indicate that the covariate effect $\beta_{2}$ and the covariate prevalence $\pi$ affect the optimal design, and the optimal design is thus sensitive to the misspecification of these two factors. The extent to which the increase of $\beta_{2}$ decreases the optimal number of time points $p^{*}$ is substantially influenced by $\pi$ : the decrease is stronger for a larger $\pi$. Similarly, the effect of $\pi$ on $p^{*}$ largely depends on $\beta_{2}$ values: $p^{*}$ increases as $\pi$ increases if $\beta_{2}<0$, whereas the reverse relation is observed if $\beta_{2}>0$ and no relation at all if $\beta_{2}=0$. The relation between the covariate and the optimal design is markedly influenced by the cost function. For example, if $\beta_{2}=-1.5, p^{*}$ increases if $\pi$ increases based on any cost function, but the increase is much stronger with cost function I.

To find the optimal design, researchers always need to make an educated guess about the model parameters based on findings in the literature or a pilot study. However, another alternative approach might be a Bayesian optimal design or a maximin optimal criterion. Applying the maximin criterion can obtain a reasonably efficient maximin design if pre-specified regions for the parameters $\beta_{1}$ and $\beta_{2}$ are restrictive, in particular if $\pi>0.1$ or $\beta_{2}<0$.

The optimal combination of the number of subjects and the measurements for each subject in longitudinal trials with discrete-time survival data has been studied by Jóźwiak and Moerbeek (2012). In general, they conclude that if the costs of obtaining one measurement are considerably lower than the costs of sampling one subject, it is wiser to sample fewer 
subjects and take more measurements. They also discuss which design factors (including $\beta_{1}, \omega, f$ and $\tau$ ) affect the optimal design and to what extent. Our study generally confirms their findings and provides additional evidence of the effect of covariate adjustment on the optimal design for a treatment effect estimator. So a design that is optimal for a positive covariate effect might be far from optimal if the covariate effect is indeed negative. Accordingly, we advise researchers to carefully specify the covariate effect and prevalence before conducting their trials.

Our findings are based on the assumptions that the treatment groups are of equal size and there is a constant attrition rate across the time periods and all the cells. However, our $\mathrm{R}$ syntax can be used in studies where the attrition rate varies across the time points and cells. From a financial as well as an ethical point of view, some longitudinal studies need to have unequal treatment groups. It might be worthwhile to investigate the extent to which the results of this study apply to longitudinal trials with unequal allocation ratio. We also assume equally spaced time periods for ease of comparison between the baseline hazard probabilities across the time periods. In practice, however, researchers might plan their designs using nonequally spaced time periods, especially in the case of a non-constant hazard function. So a decision needs to be made on the optimal number of time intervals and the optimal length of the intervals. This topic has been studied as regards quantitative outcomes since the 1990s (Tan and Berger, 1999). Another line of research would be to study the optimal number of time points and their optimal allocation for discrete-time survival analysis; efficient estimates of the baseline hazard function are also of great importance.

To our knowledge, this is the first study on the relation between a predictive covariate and optimal designs for trials with discrete-time survival endpoints. We hope our results help researchers design their trials efficiently by considering a strongly predictive covariate. Our R syntax is available in the supplementary material (see Appendix A).

\section{Acknowledgment}

This research was funded by a VIDI grant from the Netherlands Organization for Scientific Research (NWO) number 452-08-004.

\section{Appendix A. Supplementary material}

Supplementary material related to this article can be found online at http://dx.doi.org/10.1016/j.csda.2014.02.012.

\section{References}

Allison, D., 1995. When is it worth measuring a covariate in a randomized clinical trial? J. Consult. Clin. Psychol. 63, 339-343.

Allison, D., Allison, R., Faith, M., Paultre, F., Pi-Sunyer, F., 1997. Power and money: designing statistically powerful studies while minimizing financial costs. Psychol. Methods 2, 20-33.

Berger, M.P.F., Wong, W.K., 2009. An Introduction to Optimal Designs for Social and Biomedical Research. John Wiley and Sons, Ltd.

Capaldi, D.M., Crosby, J., Stoolmiller, M., 1996. Predicting the timing of first sexual intercourse for at-risk adolescent males. Child Dev. 67, $344-359$.

Galbraith, S., Marschner, I.C., 2002. Guidelines for the design of clinical trials with longitudinal outcomes. Control. Clin. Trials 23, 257-273.

Hedeker, D., Gibbons, R.D., Waternaux, C., 1999. Sample size estimation for longitudinal designs with attrition: comparing time-related contrasts between two groups. J. Educ. Behav. Stat. 24, 70-93.

Jóźwiak, K. Moerbeek, M. 2012. Cost-effective designs for trials with discrete-time survival endpoints. Comput. Statist. Data Anal. 56, $2086-2096$.

Liu, A., Boyett, J.M., Xiong, X., 2000. Sample size calculation for planning group sequential longitudinal trials. Stat. Med. 19, 205-220.

McCullagh, R.M., Nelder, J.A., 1989. Generalized Linear Models. Chapman and Hall, London.

Moerbeek, M., 2006. Power and money in cluster randomized trials: when is it worth measuring a covariate? Stat. Med. 25, $2607-2617$.

Moerbeek, M., 2008. Powerful and cost-efficient designs for longitudinal intervention studies with two treatment groups. J. Educ. Behav. Stat. 33, 41-61.

Moerbeek, M., 2012. Sample size issues for cluster randomized trials with discrete-time survival endpoints. Methodol.: Eur. J. Res. Methods Behav. Soc. Sci. $8,146-158$

Passos, V., Tan, F., Berger, M., 2011. Cost-efficiency considerations in the choice of a microarray platform for time course experimental designs. Comput. Statist. Data Anal. 55, 944-954.

Raudenbush, S.W., Liu, X.F., 2001. Effects of study duration, frequency of observation, and sample size on power in studies of group differences in polynomial change. Psychol. Methods 6, 387-401.

Safarkhani, M., Moerbeek, M., 2013. Covariate adjustment strategy increases power in the randomized controlled trial with discrete-time survival endpoints. J. Educ. Behav. Stat. 38, 355-380.

Singer, J., Willett, J., 1993. It's about time: using discrete-time survival analysis to study duration and the timing of events. J. Educ. Behav. Stat. 18, 155-195. Singer, J., Willett, J., 2003. Applied Longitudinal Data Analysis. Modeling Change and Event Occurrence. Oxford Unversity Press, Oxford.

Tan, F.E.S., Berger, M.P.F., 1999. Optimal allocation of time points for the random effects model. Comm. Statist. Simulation Comput. 28, 517-540.

Tekle, F., Tan, F., Berger, M., 2008. D-optimal cohort designs for linear mixed-effects model. Stat. Med. 27, 2586-2600.

Yi, Q., Panzarella, T., 2002. Estimating sample size for tests on trends across repeated measurements with missing data based on the interaction term in a mixed model. Control. Clin. Trials 23, 481-496. 